\title{
Influence of Surface Morphology on Absorptivity of Light-Absorbing Materials
}

\author{
Chunhui Niu $(\mathbb{D}$, Ting Zhu $\mathbb{D}$, and Yong Lv $\mathbb{D}$ \\ School of Instrument Science and Opto-Electronics Engineering, Beijing Information Science \& Technology University, \\ Beijing 100192, China \\ Correspondence should be addressed to Yong Lv; lvyong@bistu.edu.cn
}

Received 28 March 2019; Accepted 30 May 2019; Published 8 September 2019

Academic Editor: Jiangbo Yu

Copyright (C) 2019 Chunhui Niu et al. This is an open access article distributed under the Creative Commons Attribution License, which permits unrestricted use, distribution, and reproduction in any medium, provided the original work is properly cited.

\begin{abstract}
Absorptivity of three kinds of surface morphology, i.e., V-type groove surface, sinusoidal surface, and random distribution, is investigated using a rigorous electromagnetic theory and a finite element method. Influences of surface contour parameters (span distance, intersection angle, and height) and light wave parameters (incident angle and wavelength) on absorptivity are numerically simulated and analyzed for the three kinds of surfaces, respectively. Absorbing spectra about three silicon wafers with different surface roughness are recorded, and the results are coincident with simulated results.
\end{abstract}

\section{Introduction}

Improving absorptivity of light-absorbing materials is very significant in laser machining, solar cell manufacturing, and light-sensitive detector fields, wherein surface morphology and surface roughness become one major factor of influencing material absorptivity. When heat treatment is with a laser beam, a metallic material with a smooth surface has only less than ten percent absorptivity to laser [1], and absorptivity of nonmetallic materials relates to the incident angle of laser beam, which is affected by material surface roughness $[2,3]$. For metallic and nonmetallic materials, its absorptivity can be heightened through adjusting material surface roughness due to increased light reflected and absorbed a number of times. Solar cell can transfer radiation energy to electrical energy, and its absorptivity is one of the most important performance parameters. Many methods can be used to enhance absorptivity to solar light, e.g., overlaying a surface layer with high absorptivity [4-6], doping with metal nanoparticles to motivate surface plasma polarization excimer [7-9], and controlling surface morphology [10-14]; here, improving surface roughness is a very effective method. Furthermore, to improve the performance of a solar radiometer, some black coating material with high absorptivity is overplayed on the surface and its surface roughness is changed [15].
Therefore, investigating the relationship between material absorptivity and surface roughness of light-absorbing materials is significant.

Li et al. [15] and Su et al. [16] have analyzed the light absorbing ability of several materials with different types of a surface morphology-based light ray tracing method and achieved some common characteristics of improving light absorptivity through changing surface morphology and derived a relationship formula between absorptivity and surface morphology parameters. Chen et al. [17] have equivalently dealt with surface contour line and established a computational model of influence of surface roughness on laser absorptivity using a light ray tracing method and provided a more convenient method for calculating laser absorptivity in a laser heat treatment field.

However, the light ray tracing method is based on a hypothesis that light propagates along a straight line, which is only valid when the characteristic structure dimension of surface morphology of absorbing material, e.g., the period of a microstructure and span of random fluctuation, is far greater than incident light wavelength. But the majority of surface random fluctuations of a common material belong to a micrometer magnitude order and are close to visible light wavelength; therefore, the light ray tracing method is not suited to calculate the absorptivity of a rough surface. 
In this thesis, a rigorous electromagnetic theory is adopted to analyze the influence of surface morphology and surface roughness on material absorptivity. COMSOL Multiphysics, which is a commercial software and can be utilized to numerically solve partial differential equations based on a finite element analysis method, is used to solve Maxwell's equations and simulate the absorbing effect of the rough surface to light. The absorptivity of the three kinds of surface contour profiles such as the V-type groove structure, sinusoidal wave structure, and random fluctuation structure is numerically calculated, and relationship curves between light absorbing performance and surface morphology parameters are obtained. At last, absorbing spectra of three silicon wafers with different surface roughness are measured, and the results are basically consistent with the numerical simulation results.

\section{Shortcoming of a Light Ray Tracing Method}

Optical wave is a kind of electromagnetic wave in nature, and its propagation obeys Maxwell's equations, which can be expressed as

$$
\begin{aligned}
\nabla \times \mathbf{H} & =\mathbf{J}+\frac{\partial \mathbf{D}}{\partial t}, \\
\nabla \times \mathbf{E} & =-\frac{\partial \mathbf{B}}{\partial t}, \\
\nabla \cdot \mathbf{D} & =\rho, \\
\nabla \cdot \mathbf{B} & =0,
\end{aligned}
$$

where $\mathbf{E}$ and $\mathbf{D}$ represent the electric intensity vector and electric displacement vector, respectively, and have a relationship as $\mathbf{D}=\varepsilon \mathbf{E}$, where $\varepsilon$ is the dielectric constant. $\mathbf{H}$ and $\mathbf{B}$ are the magnetic intensity vector and magnetic induction intensity vector, respectively, and have a relationship as $\mathbf{B}=\mu \mathbf{H}$, where $\mu$ is the magnetic permittivity. Furthermore, $\mathbf{J}$ is the current density, and $\mathbf{J}=\sigma \mathbf{E}$, where $\sigma$ represents the electrical conductivity.

A harmonic plane wave can be expressed as

$$
\begin{aligned}
\mathbf{E}(\mathbf{r}, \mathbf{t}) & =\mathbf{E}(\mathbf{r}) e^{j \omega t}, \\
\mathbf{H}(\mathbf{r}, \mathbf{t}) & =\mathbf{H}(\mathbf{r}) e^{j \omega t}, \\
\mathbf{E}(\mathbf{r}) & =\mathbf{e}(\mathbf{r}) e^{j k_{0} S(\mathbf{r})}, \\
\mathbf{E}(\mathbf{r}) & =\mathbf{e}(\mathbf{r}) e^{j k_{0} S(\mathbf{r}),}
\end{aligned}
$$

where $\omega$ is the circular frequency, $k_{0}$ depicts the wave vector in vacuum, and $S(\mathbf{r})$ represents the optical path length and is a real scalar function.

Supposing that light wavelength $\lambda$ can be approximated to an infinitely small quantity, an equation can be deduced as $\nabla S(\mathbf{r})=n$, where $n$ represents the refractive index of propagation medium. For an isotropic and homogeneous medium, it can be derived from the equation $\nabla \mathrm{S}(\mathbf{r})=n$ that light wave propagates along a straight line.



FIgURE 1: Schematic diagram of light incident on an absorption material.

As mentioned above, the light ray tracing method is valid only when three conditions are satisfied: (1) a harmonic wave, (2) $\lambda \rightarrow 0$, and (3) $n=$ constant.

If a monochromatic plane wave is incident on a material with a smooth and infinite surface, as shown in Figure 1, according to the Fresnel formula [18], the reflectance can be expressed as

$$
\rho=\frac{1}{2}\left[\frac{(n-\cos \alpha)^{2}+k^{2}}{(n+\cos \alpha)^{2}+k^{2}}+\frac{(n-(1 / \cos \alpha))^{2}+k^{2}}{(n+(1 / \cos \alpha))^{2}+k^{2}}\right],
$$

where $n$ is the material refractive index, $k$ is the light extinction coefficient, and $\alpha$ is the incident angle. If the thickness of the absorbing material is enough to absorb all the transmission light, absorptivity of the absorption material to the incident light can be expressed as $\mathrm{A}(\alpha)=1-\rho$.

A V-type groove structure is always used to replace the surface contour profile of a naturally formed and processed coarse surface. According to the light ray tracing method, an incident light beam will endure many times the reflection in a V-type groove, as shown in Figure 2(a). In Figure 2(a), $L$ represents the opening width, $H$ is the depth of a $\mathrm{V}$-type groove, and $\theta$ is an intersection angle, and there is a relationship formula: $L=2 H \bullet \sin (\theta / 2)$. The whole absorbing percentage of light in a $\mathrm{V}$-type groove can be obtain by considering reflectance in every time according to equation $(3)[15,16]$.

As mentioned above, the light ray tracing method can be adopted only when the opening width $L$ of the $\mathrm{V}$-type groove is far greater than incident light wavelength $\lambda$. If $L$ is approximately equal to or less than $\lambda$, the diffraction of the V-type groove and interference among reflecting wave must be considered. In this moment, the light field distribution will be complex and must be calculated through a rigorous electromagnetic theory.

In Figure 2(b), the electric intensity $|E|^{2}$ distribution for a V-type groove structure with $L=2 \mu \mathrm{m}, \theta=60^{\circ}$, and $\lambda=0.8 \mu \mathrm{m}$ is shown. It can be seen from Figure 2(b) that, due to considering many time reflection, diffraction, and multiple-beam interference in the rigorous electromagnetic theory, the last light field distribution is inhomogeneous, but the result cannot be obtained through the light ray tracing method. 


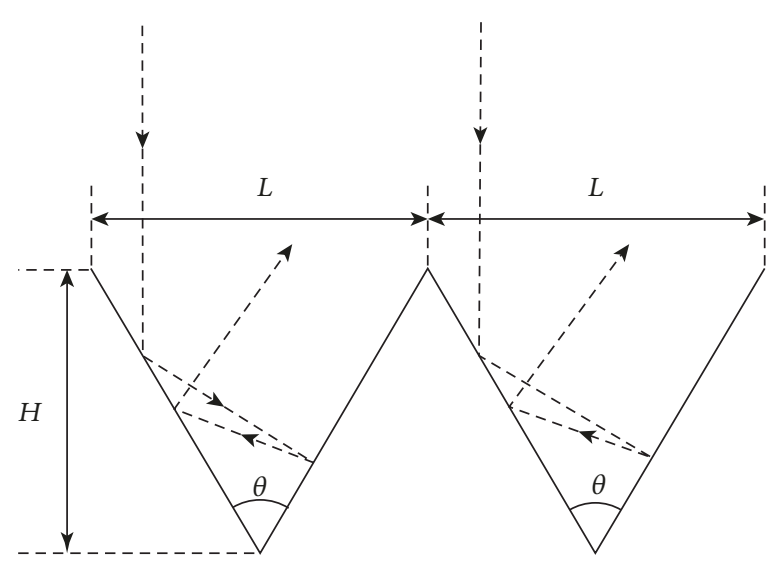

(a) Light ray tracing



(b) $|E|^{2}$ calculated through the finite element method

Figure 2: Surface structure of V-type groove and light field distribution.

\section{Simulation Results and Analysis Based upon a Rigorous Electromagnetic Theory}

In order to analyze the influence of surface morphology on absorptivity more comprehensively, two types of surface contour profiles are adopted, that is, regular surface morphology and random surface morphology. Here, regular surface morphology includes the $\mathrm{V}$-type groove structure and sinusoidal structure, which are two-dimension symmetry and elementary shape functions and often used to replace contour profiles of coarse surfaces. A random surface has random height values with normal distribution and is more similar to realistic surface morphology.

Figure 3 shows three kinds of different surface morphologies, which are the V-type groove structure, sinusoidal structure, and random structure.

The absorbing performances of different surface morphologies are numerically simulated and analyzed by adopting the wave optics module in commercial software COMSOL Multiphysics, which is based upon the finite element method to solve Maxwell's equations.

Simulation parameters are listed in Table 1. For convenience, a 2-dimension structure is considered and the simulation region sizes are $10 \mu \mathrm{m}$ in the horizontal direction and $10 \mu \mathrm{m}$ in the vertical direction. The left and right boundaries adopt the periodic boundary condition, and the up and down boundaries use the "port" boundary condition. The light is incident from air to silicon substrate. In order to investigate the general absorption rule, the refractive index and extinction coefficient of air and silicon subtracts are changeless for all simulated light wavelength.

Figure 4 shows the mesh subdivision diagram for the three kinds of surface structures, wherein the upper half is air and the lower half is crystalline silicon. In order to guarantee the accuracy of the solution, the biggest mesh dimension is less than $\lambda_{0} / 6 \bullet n$, where $\lambda_{0}$ represents the incident light wavelength in vacuum and $n$ is the refractive index of air or crystalline silicon.
3.1. Absorptivity vs. Surface Morphology Parameters of a $V$-Type Groove Structure. Figure 5 displays the absorbing performance of the $\mathrm{V}$-type groove surface structure. In this case, light wave with wavelength $\lambda=0.8 \mu \mathrm{m}$ is normally incident on the crystalline silicon surface with the $\mathrm{V}$-type groove as two electromagnetic polarization modes: TE mode ( $\mathbf{E}$ vector is perpendicular to the water plane) and TM mode ( $\mathbf{H}$ vector is perpendicular to the water plane). In Figure 5, the $x$-axis represents the intersection angle of the $\mathrm{V}$-type groove and the $y$-axis represents absorptivity.

It can be seen from Figure 5 that, for two opening widths $L=0.5 \mu \mathrm{m}$ and $L=1 \mu \mathrm{m}$, absorptivity corresponding to the TM mode is larger than absorptivity to the TE mode, which is because in this simulation a two-dimension structure is adopted, i.e., the $\mathrm{V}$-type groove structure is changeless along the $y$-axis, so that there exists polarization selectivity. However, for the three-dimension V-type groove structure, as shown in Figure 3, the absorbing performance should be mixed results by the TE mode and TM mode. Therefore, in subsequent discussion, the total absorptivity will be an average value of the TE mode and TM mode.

Furthermore, it is obvious in Figure 5 that four absorptivity curves roughly tend to decrease along with the increasing angle $\theta$, which is similar to results according to the light ray tracing method $[15,16]$. But when the angle $\theta$ is less, absorptivity curves have some fluctuation. The appearance can be explained as the light wave incident on the $\mathrm{V}$-type groove surface with less angle $\theta$ will endure more reflecting times and there exist more complex multiple-beam interference results, but for the case $\theta>120^{\circ}$, light wave is only reflected once, so that absorptivity monotonously decreases according to the analysis of equation (3).

In addition, it can also be seen from Figure 5 that, for the identical intersection angle $\theta$, absorptivity corresponding to opening width $L=1 \mu \mathrm{m}$ is larger than absorptivity to $L=0.5 \mu \mathrm{m}$, which is because when $\theta$ is a constant quantity, a larger opening width means a deeper $\mathrm{V}$-type groove and more light reflection times. 




(a) V-type groove structure

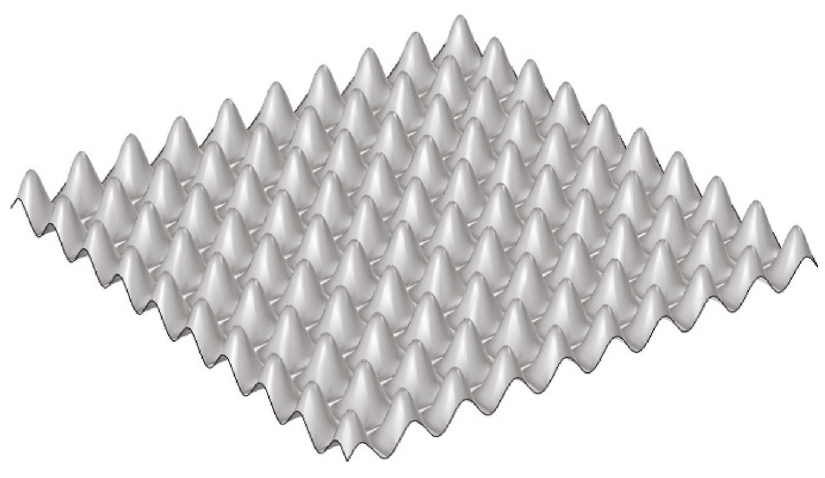

(b) Sinusoidal structure



(c) Random structure

FIgURE 3: Three kinds of surface morphology.

TABLE 1: Simulation parameters.

\begin{tabular}{lcc}
\hline \multicolumn{2}{c}{ Parameters } & Value \\
\hline Region size & Horizontal & $10 \mu \mathrm{m}$ \\
Vertical & $10 \mu \mathrm{m}$ \\
\hline Boundary condition & Left-right & $\begin{array}{c}\text { Periodic boundary } \\
\text { condition } \\
\text { Port boundary } \\
\text { condition }\end{array}$ \\
\hline \multirow{2}{*}{ Mesh size } & Up-down & $\frac{\lambda_{0}}{6 \bullet n}$ \\
& The biggest & $5 \mathrm{~nm}$ \\
Air & The least & 1 \\
& Refractive index & 0 \\
Si & Extinction coefficient & 4.2 \\
& Refractive index & 0.06 \\
\hline
\end{tabular}

In the light ray tracing method, suppose $\lambda \rightarrow 0$, light absorptivity is independent of light wavelength. But according to the rigorous electromagnetic theory, light propagation performance in medium is influenced by light wavelength deeply. Figure 6 shows the absorptivity varying curve of the V-type groove structure along with light wavelength when $\theta=60^{\circ}, L=1 \mu \mathrm{m}$, and $\alpha=0^{\circ}$. Within the wavelength scope from $0.4 \mu \mathrm{m}$ to $1 \mu \mathrm{m}$, absorptivity has only weak variety, but when light wavelength continues to increase, absorptivity will tend to decrease, and the absorptivity curve has more and more fluctuation. This result can be explained as follows: when the opening width $L$ of the $\mathrm{V}$-type groove is larger than light wavelength, the light wave can be treated approximately as light beam with a straight propagation path, but when $L<\lambda$ and $H<\lambda$, the $\mathrm{V}$-type groove surface can be taken as an equivalent homogeneous medium layer $[19,20]$; the reflectivity will fluctuate along with increasing light wavelength due to multiple-beam interference of the equivalent film, and at the same time, larger light wavelength means thinner film thickness and weaker absorbing ability.

Figure 7 shows the absorptivity varying curve along with the light incident angle $\alpha$ when $L=1 \mu \mathrm{m}$ and $\lambda=$ $0.8 \mu \mathrm{m}$. It is obvious that in three curves, absorptivity roughly reduces with the incident angle $\alpha$ increasing, but a larger intersection angle $\theta$ corresponds to a larger fluctuation of the absorptivity curve. This appearance can be explained as follows: when the intersection angle $\theta$ is small, light wave will be reflected many times in the V-type groove, so that reflecting times reducing caused by the light incident angle $\alpha$ changing can be neglected; but when $\theta$ is larger, light wave only endures less reflecting times in the V-type groove, reflecting times changing with the incident angle will be very dominant. 


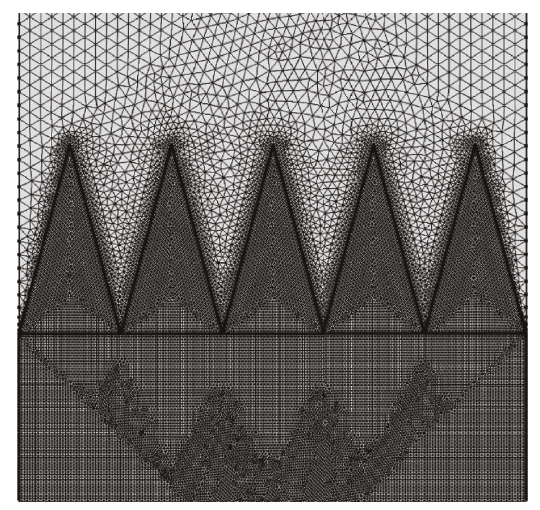

(a) V-type groove structure



(b) Sinusoidal structure

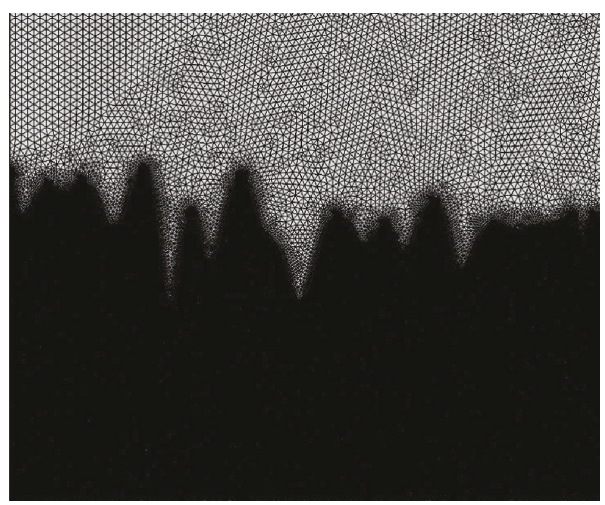

(c) Random structure

FIGURE 4: Mesh subdivision diagram of three surface structures.

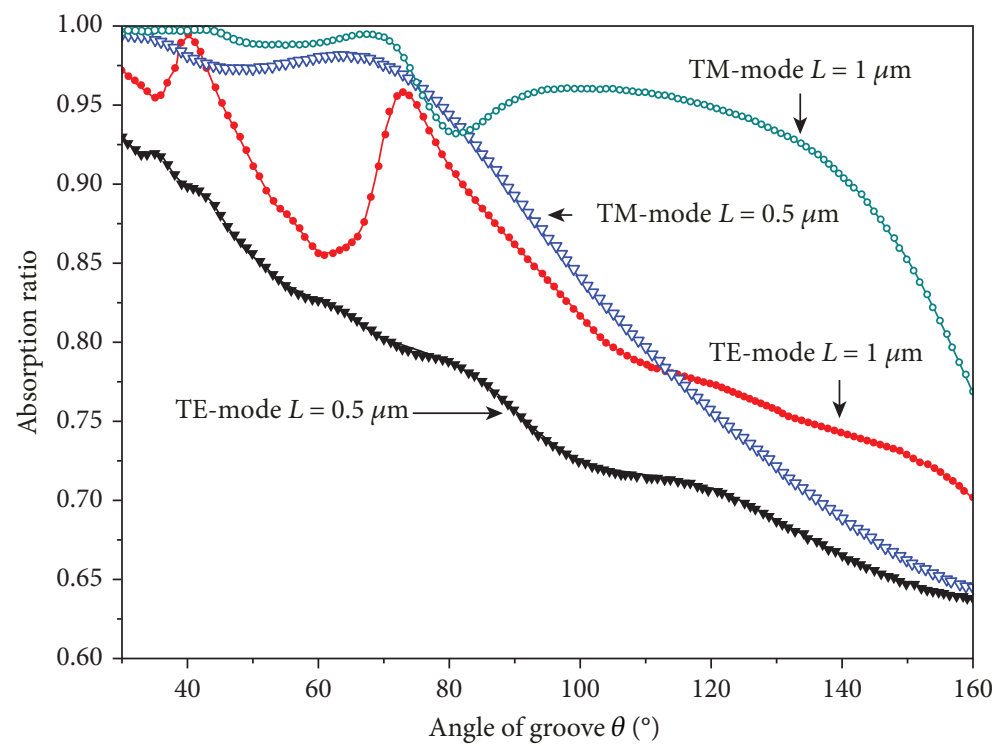

FIGURE 5: Varying curve of absorptivity along with the intersection angle of the V-type groove structure.

3.2. Absorptivity vs. Surface Morphology Parameters of a Sinusoidal Surface Structure. The sinusoidal surface structure can be looked as a passivated V-type groove structure, and its equivalent intersection angle $\theta$ can be expressed as $\theta=2$ $\arctan (T / 4 A)$, where $T$ means the period of the sinusoidal surface structure and $A$ is the amplitude, as shown in Figure 8.

Figure 9 shows the absorptivity varying curve along with light wavelength supposing that $T=1 \mu \mathrm{m}, \theta=60^{\circ}$, and $\alpha=0^{\circ}$. 




FIGURE 6: Absorptivity varying curve of V-type groove structure along with wavelength.



FIGURE 7: Absorptivity varying curve along with incident angle.

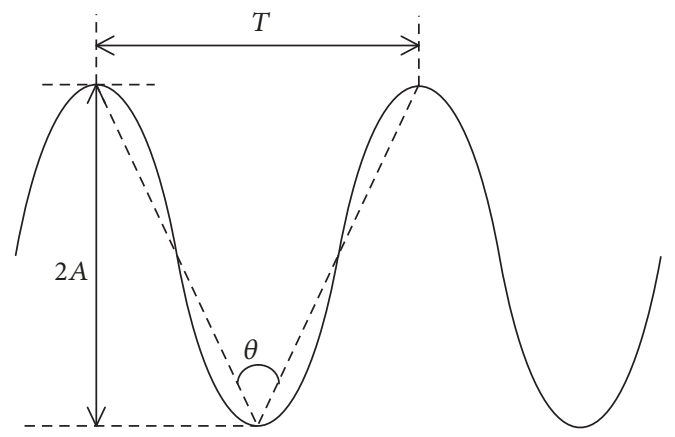

Figure 8: Sinusoidal surface structure.



FIGURE 9: Absorptivity varying curve of sinusoidal surface structure along with wavelength.



FIgURE 10: Absorptivity varying curve of sinusoidal surface structure along with incident angle.

It can be seen from Figure 9 that in the whole scope from $0.4 \mu \mathrm{m}$ to $4 \mu \mathrm{m}$, there exists a distinct fluctuation but absorptivity decreases with increasing light wavelength, approximately.

Figure 10 shows the absorptivity varying curve of the sinusoidal surface structure along with the light incident angle $\alpha$ when $T=1 \mu \mathrm{m}$ and $\lambda=0.8 \mu \mathrm{m}$. It is very similar to Figure 7 that absorptivity approximately reduces with the increasing incident angle $\alpha$, but the larger intersection angle $\theta$ corresponds to the larger fluctuation of the absorptivity curve.

3.3. Absorptivity vs. Surface Morphology Parameters of a Random Surface with Normal Distribution. The height of a common realistic structure of a natural or treated surface is 


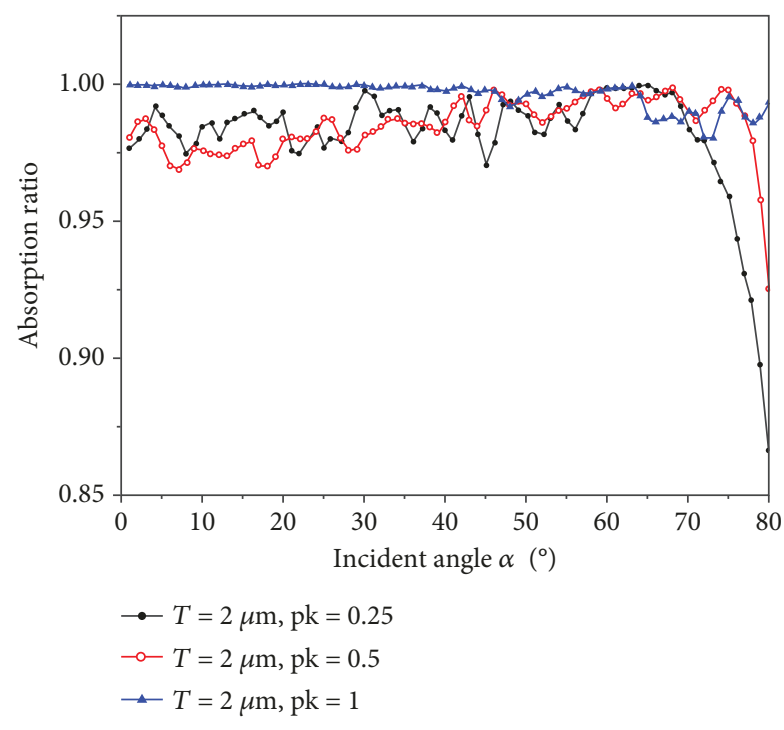

FIGURE 11: Absorptivity varying curve of random surface structure.

often normal random distribution, in which height probability density function can be expressed as

$$
p(z)=\frac{\mathrm{pk}}{\sqrt{2 \pi \sigma}} \exp \left[-\frac{(z-\mu)^{2}}{2 \sigma^{2}}\right],
$$

where $p(z)$ represents the probability density of the surface height, pk is the height scale coefficient, and $\mu$ and $\sigma$ are the mean value and root mean square value (RMS) of the surface height, respectively. In this thesis, a normal-distribution random surface with $\mu=0$ and $\sigma=1 \mu \mathrm{m}$ is adopted.

Figure 11 illustrates the absorptivity varying curve of the random surface structure with different pk values, where $T$ is defined as the span distance between two wave peaks in the random distribution surface and $T=2 \mu \mathrm{m}$. It is clearly seen that when $\mathrm{pk}=0.25$ and $\mathrm{pk}=0.5$, there are obvious fluctuations in the absorptivity varying curve, but when $\mathrm{pk}=1$, absorptivity is close to 1 and there are only small fluctuations. A larger pk value means a higher groove depth and a small intersection angle, similar to cases in Figures 7 and 10, so that when roughness is high enough, the absorptivity of the coarse surface will be independent to the light incident angle.

The absorptivity varying curve of the random surface structure with $T$ taking different values is shown in Figure 12 . When the light incident angle is less than $60^{\circ}$, it is very clear that a smaller span distance $T$ value corresponds to a higher absorption ratio, but for an incident angle bigger than $60^{\circ}$, there are larger fluctuations in absorptivity varying curve and there exists no obvious tendency with a changing $T$ value. In most cases, light wave is incident with an incident angle less than $60^{\circ}$; an identical pk value and a smaller $T$ value, corresponding to higher surface roughness, will have a higher absorption ratio.

Figure 13 shows the absorptivity varying curve of the random surface structure along with light wavelength supposing that $T=2 \mu \mathrm{m}, \mathrm{pk}=1$, and $\alpha=0^{\circ}$. It can be seen that in the whole scope from $0.4 \mu \mathrm{m}$ to $4 \mu \mathrm{m}$, absorptivity decreases with

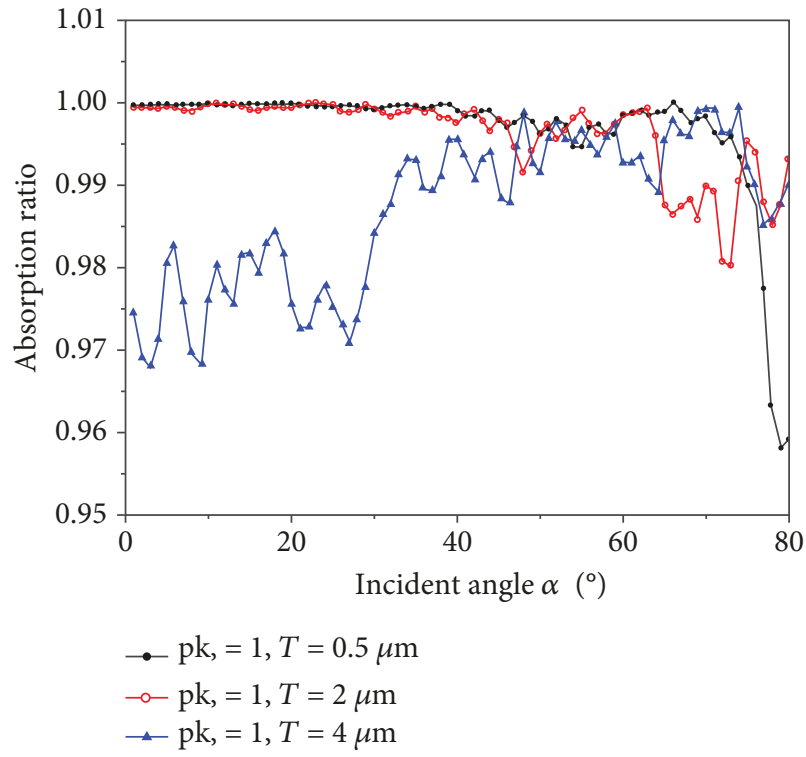

FIGURE 12: Absorptivity varying curve of random surface structure with different $T$ values.

light wave increasing approximately but with some fluctuation, the same as in Figure 9.

According to the above analysis, for improving the absorption ability of silicon subtract, the random surface structure should have a larger pk value and a smaller $T$ value, the suitable regions of the ratio $\mathrm{pk} / T$ are from 0.5 to 10 , because a larger $\mathrm{pk} / T$ value is difficult to realize at the present technological level. Moreover, in the above simulation, the extinction coefficient of silicon substrate is changeless for different light wavelengths, but in fact, crystalline silicon material has larger absorption ability in a visible light region but lower absorption in a near-infrared light region. In consideration of the above simulation results and absorption characteristic of silicon material, the suitable wavelength region is from $400 \mathrm{~nm}$ to $600 \mathrm{~nm}$.

\section{Experimental Verification}

In order to verify the effectiveness of the above simulation and analyzed results, the three kinds of coarse surfaces of silicon wafer are considered to investigate absorbing ability.

The roughness of the three surfaces is measured by using a Talyrond-365 type of a surface roughometer produced by Taylor Hobson Ltd. The measured roughness curves are shown in Figure 14.

In Figure 14, $y$-axis represents random fluctuation and its unit is micrometer. Three surface roughness curves are all random distribution and belong to the three kinds of surfaces, i.e., the smooth front surface, the rough rear surface, and the treated rear surface of a commercial silicon wafer, respectively. The contour arithmetic mean $R_{\mathrm{a}}$ is often used to estimate the roughness level of the coarse surface, and $R_{\mathrm{a}}$ of three surfaces shown in Figure 14 are $0.0153 \mu \mathrm{m}$, $0.9994 \mu \mathrm{m}$, and $0.9038 \mu \mathrm{m}$, respectively.

Reflection of light on the coarse surface belongs to diffuse reflection. Diffuse reflection spectra of three surfaces are 


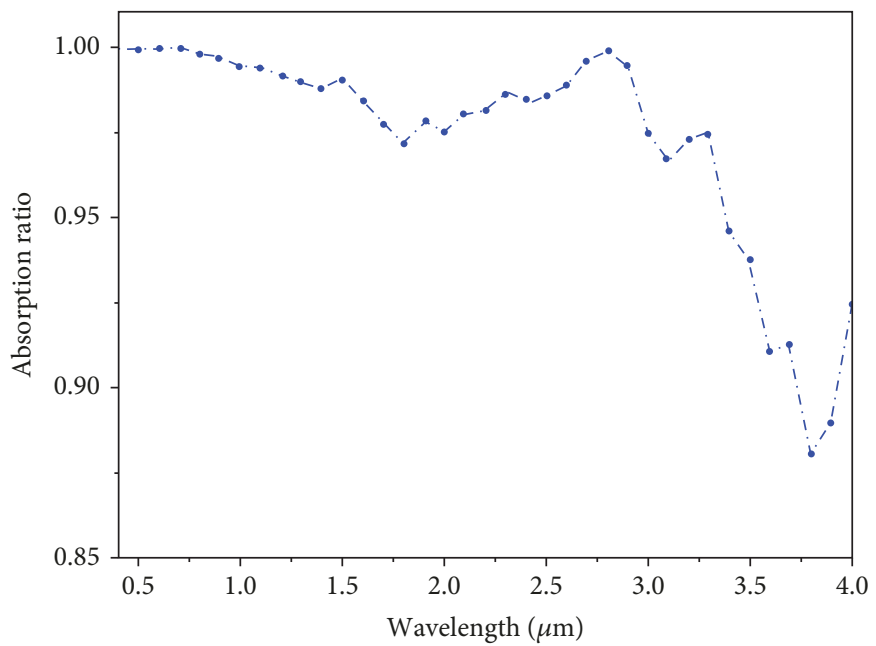

FIGURE 13: Absorptivity varying curve of random surface structure along with wavelength.
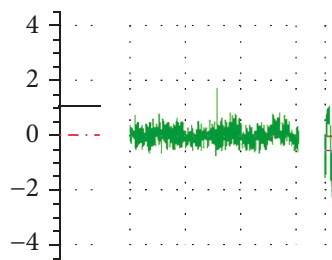

(a)



(b)



(c)

Figure 14: Roughness measured from three different surface patterns.

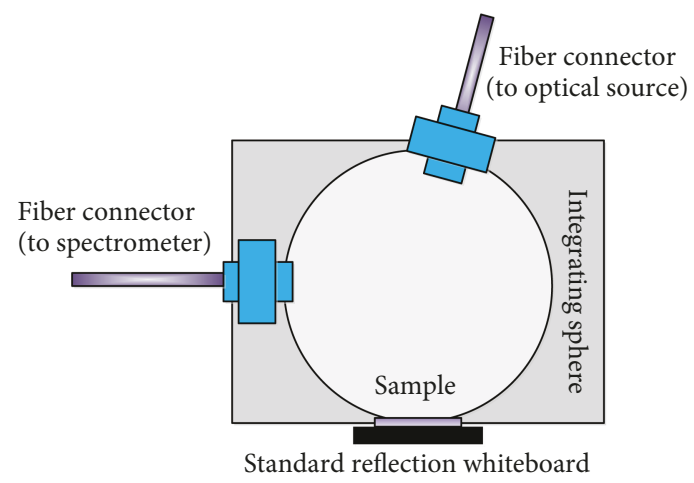

FIGURE 15: Set-up diagram for measuring diffuse reflection spectrum.

measured by using a set-up shown in Figure 15, white optical emitted from a halogen tungsten lamp (AvaLight-DHc) is guided into integrating sphere (AvaSphere-50) through an optical fiber, a part of incident white light is absorbed by measured powder sample placed above a standard reflection whiteboard, and other incident white light is scattered in all directions. The scattered light will be scattered secondly or repeatedly when collided with the wall of the integrating sphere, so after many times of collision, remaining scattered light will have spatially uniform distribution in an integrating sphere. A fiber connector is used



Figure 16: Absorption spectrum for three surfaces.

to guide the remaining part of scattered light into a fiber spectrometer (AvaSpec-ULS2048-USB2) for measuring diffuse reflection spectrum. The absorptivity of the sample can be expressed as $A(\lambda)=1-\mathrm{I}(\lambda) / I_{0}(\lambda)$, where $I(\lambda)$ represent the diffuse light intensity measured as the set-up shown in Figure 15 and $I_{0}(\lambda)$ is the diffuse light intensity measured only for a standard reflection whiteboard.

Figure 16 shows the measured absorption spectrum of the three surfaces. For the smooth surface, there is a $R_{\mathrm{a}}$ value of $0.0153 \mu \mathrm{m}$ and the lowest roughness, so it should have the lowest absorption spectrum, which is in accordance with the result shown in Figure 16. It can be seen in Figure 14 that the rough surface and the treated rough surface have close $R_{\mathrm{a}}$ values $(0.9994 \mu \mathrm{m}$ and $0.9038 \mu \mathrm{m})$, but the treated rough surface has more spacing distance $T$ or intersection angle $\theta$ than 
the rough surface; therefore, the treated rough surface should have lower absorptivity, which is also coincident with the results in Figure 16.

\section{Conclusion}

In conclusion, the absorptivity of the three kinds of surfaces with different morphologies is investigated by using the rigorous electromagnetic theory and finite element method. The three surfaces are the V-type groove surface, sinusoidal surface, and random distribution surface and are gradually close to a realistic coarse surface. For the three surface, influences of surface contour parameters (span distance, intersection angle, and height) and light wave parameters (incident angle and wavelength) on absorptivity are numerically simulated and analyzed. Moreover, absorbing spectra about three silicon wafers with different surface roughness are measured, and the results are coincident with simulated results.

\section{Data Availability}

The (figures and tables) data used to support the findings of this study are included within the article.

\section{Conflicts of Interest}

The authors declare that there is no conflict of interest regarding the publication of this paper.

\section{Acknowledgments}

The thesis is funded by the "Project of Development and Improvement of Scientific Research of Beijing Information Science and Technology University."

\section{References}

[1] J. Lu, X. W. Ni, and A. Z. He, Reciprocity between Laser and Material Physics, China Machine Press, Beijing, 1996, in Chinese.

[2] Q. H. Chen, Reciprocity between Laser and Material« Thermal Simulation, Yunnan Science and Technology Press, Kunming, 2001, (in Chinese).

[3] Y. J. Guo, C. S. Liu, and C. L. He, "89-1 coating for laser surface hardening," Laser Technology, vol. 26, p. 252, 2002.

[4] A. Roos, M. Georgson, and E. Wäckelgård, "Tin-oxide-coated anodized aluminium selective absorber surfaces I. Preparation and characterization," Solar Energy Materials, vol. 22, no. 1, pp. 15-28, 1991.

[5] A. Roos and M. Georgson, "Tin-oxide-coated anodized aluminium selective absorber surfaces II. Aging and durability," Solar Energy Materials, vol. 22, no. 1, pp. 29-41, 1991.

[6] Q. C. Zhang, "Recent progress in high-temperature solar selective coatings," Solar Energy Materials \& Solar Cells, vol. 62, no. 1-2, pp. 63-74, 2000.

[7] Y. Zhang, Z. Ouyang, N. Stokes, B. Jia, Z. Shi, and M. Gu, "Low cost and high performance Al nanoparticles for broadband light trapping in Si wafer solar cells," Applied Physics Letters, vol. 100, no. 15, p. 151101, 2012.

[8] R. A. Pala, J. White, E. Barnard, J. Liu, and M. L. Brongersma, "Design of Plasmonic Thin-Film Solar Cells with Broadband
Absorption Enhancements," Advanced Materials, vol. 21, no. 34, pp. 3504-3509, 2009.

[9] H. Y. Wang, J. X. Lu, and F. Wu, "Correlative study of porous silicon using for antireflection coating of solar cells," Journal of Synthetic Crystals, vol. 5, p. 1075, 2006.

[10] C. C. Yan, G. G. Xue, C. Liu, and S. M. Gao, "Study on the three-dimensional theory of super thin multi-layered films irradiated by the continuously modulated laser," Acta Physica Sinica, vol. 54, p. 3058, 2005.

[11] B. Q. Zhou, F. Z. Liu, M. F. Zhu, Y. Q. Zhou, Z. H. Wu, and $\mathrm{X}$. Chen, "Studies on surface roughness and growth mechanisms of microcrystalline silicon films by grazing incidence X-ray reflectivity," Acta Physica Sinica, vol. 56, p. 2422, 2007.

[12] X. C. Ren and L. X. Guo, "Investigation on the characteristics of electromagnetic scattering from 2-D fBm rough surface of layered medium," Acta Physica Sinica, vol. 58, p. 1627, 2009.

[13] D. M. Zhang, L. Li, Z. H. Li, L. Guan, S. P. Hou, and X. Y. Tan, "Variation of the target absorptance and target temperature distribution before melting in the pulsed laser ablation process," Acta Physica Sinica, vol. 54, p. 1283, 2005.

[14] Z. Ji-Yan, Y. Jia-Min, X. Yan et al., "Absorption experiments on radiatively heated Al plasma," Acta Physica Sinica, vol. 57, no. 2, pp. 985-989, 2008.

[15] L. Ya-Nan, L. Zhong-Zhu, L. Jing-Qiu et al., "Design and preparation of black-nickel film on the radiometer chip," Acta Physica Sinica, vol. 59, p. 4530, 2010.

[16] F. G. Su, J. Q. Liang, Z. Z. Liang, and W. B. Zhu, "Study on the surface morphology and absorptivity of light-absorbing materials," Acta Physica Sinica, vol. 60, article 057802, 2011.

[17] J. Chen, Q. L. Zhang, J. H. Yao, and J. B. Fu, "Influence of surface roughness on laser absorptivity," Laser Technology, vol. 32, p. 624, 2008.

[18] M. Born, E. Wolf, and A. B. Bhatia, Principles of Optics: Electromagnetic Theory of Propagation, Interference and Diffraction of Light, Cambridge University Press, Cambridge, 7th edn. edition, 2005.

[19] A. G. Lopez and H. G. Craighead, Optics Letters, vol. 23, no. 20, pp. 1627-1629, 1998.

[20] D. L. Brundrett, E. N. Glytsis, and T. K. Gaylord, "Normalincidence guided-mode resonant grating filters:?design and experimental demonstration," Optics Letters, vol. 23, no. 9, pp. 700-702, 1998. 

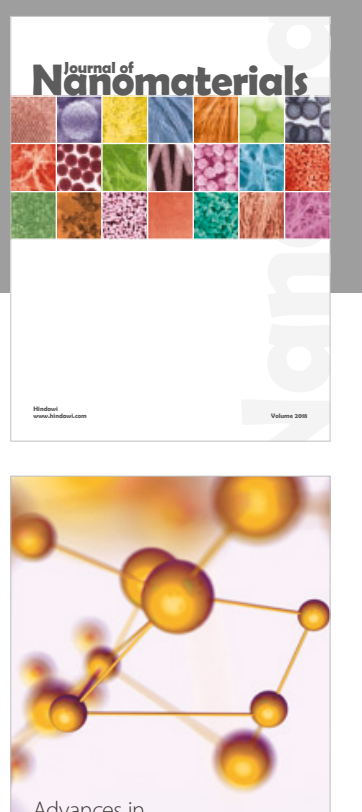

Physical Chemistry
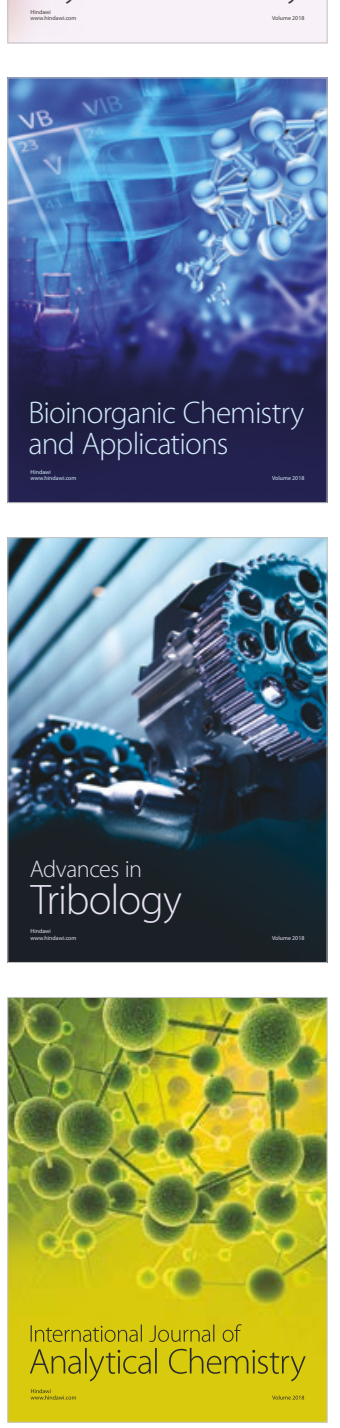

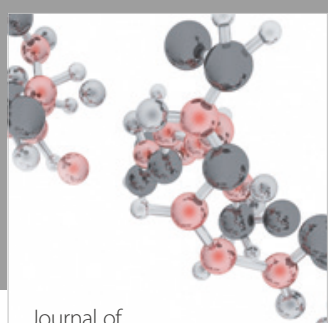

Analytical Methods

in Chemistry

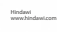

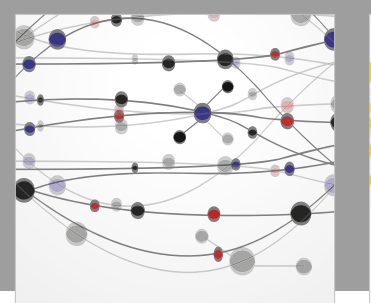

The Scientific World Journal

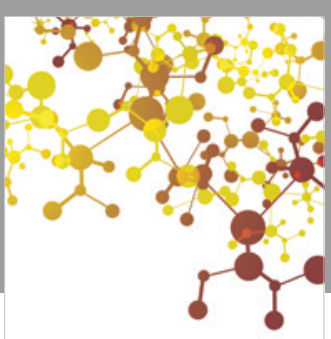

Journal of

Applied Chemistry


Submit your manuscripts at

www.hindawi.com
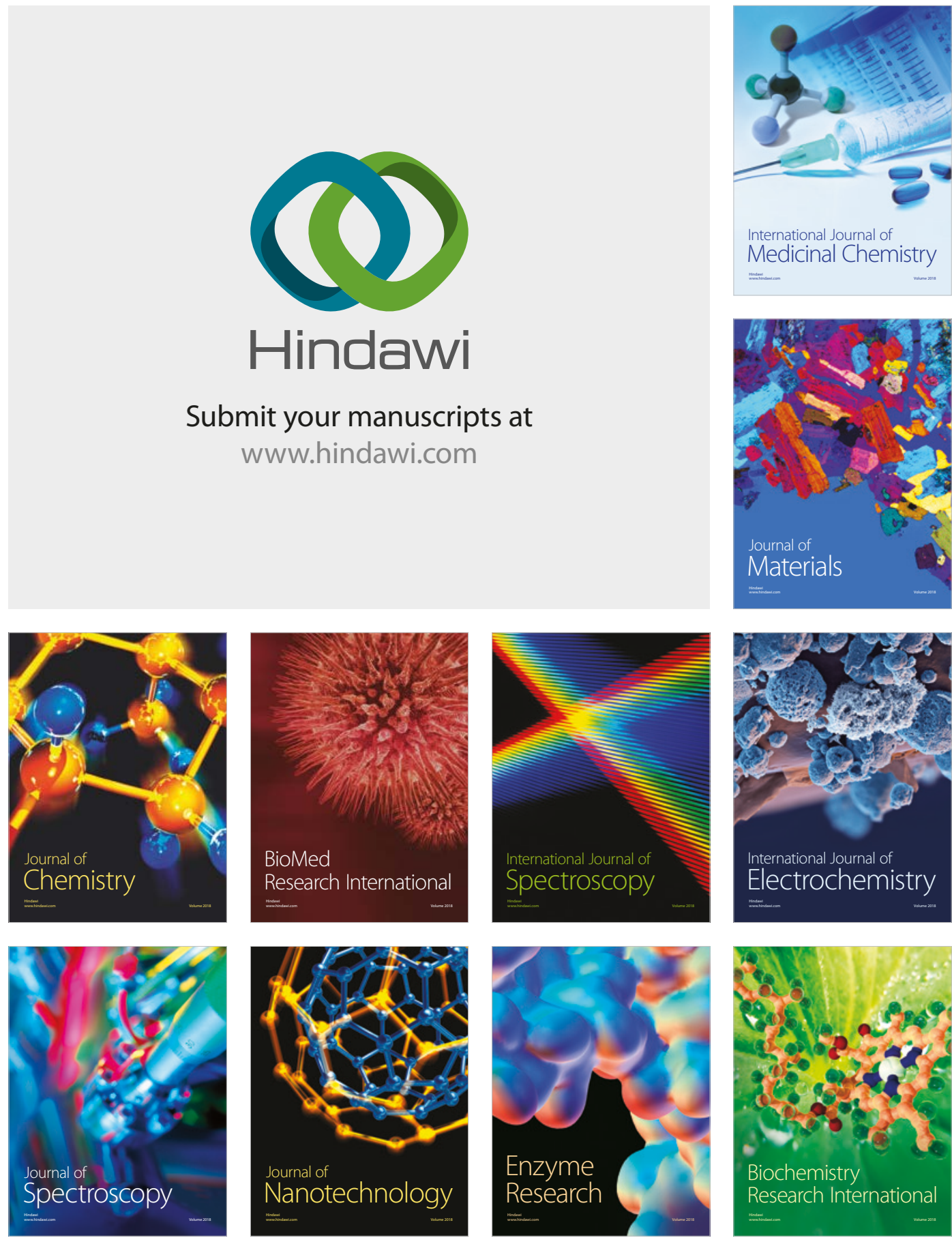
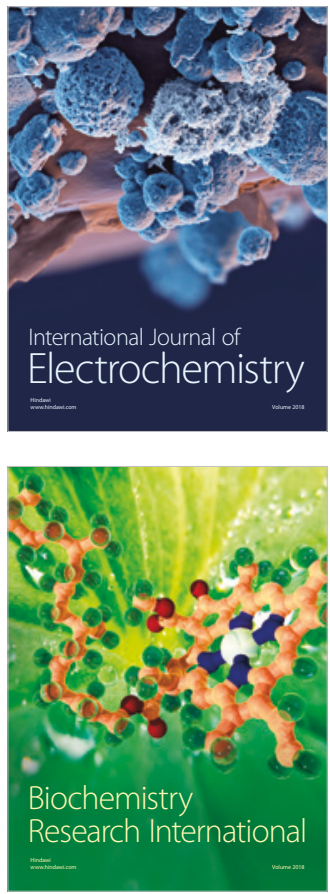